\title{
Central Nervous System Involvement in Hodgkin's Lymphoma Associated With Epstein-Barr Virus in a Patient With AIDS: Case Report and Review of the Literature
}

\author{
Marcelo Corti, María F. Villafañe Fioti, \\ Claudio Yampolsky, Ricardo Schtirbu and \\ Marina Narbaitz
}

\author{
Division of HIVIAIDS, Neurosurgery and Histopathology Laboratory, \\ Infectious Diseases F.J. Muñiz Hospital and Histopathology Laboratory, \\ National Academy of Medicine, Buenos Aires, Argentina
}

\begin{abstract}
Intracranial and intraspinal involvement is a rare complication of Hodgkin's disease. We report a case of a patient with diagnosis of AIDS and Hodgkin's lymphoma who developed brain and spinal involvement at the time of the relapse of the neoplasm disease. Mixed cellularity histology was the subtype of Hodgkin's disease in our patient; we identified the Epstein-Barr virus genome in the Reed-Sternberg cells by immunohistochemistry and in situ hybridization. Key Words: Hodgkin lymphoma, central nervous system, AIDS.
\end{abstract}

Hodgkin's lymphoma (HL) involving the central nervous system (CNS) is extremely rare [1]. Involvement of the brain or spinal cord by HL can be primary or, more frequently, secondary to the compromise of adjacent lymph nodes. Lesions are more frequently intraspinal than intracranial [2].

We report a clinical course of a patient with advanced AIDS/HIV-1 disease who developed HL complicated by CNS involvement with intracranial and spinal cord relapse.

\section{Case Report}

A 29-year-old man, intravenous drug abuser, HIV-1 seropositive with diagnosis of AIDS since 1992, was admitted to our hospital in June 2001, presenting fever, continual headaches and spastic paraparesis during 10 days. He had a history of HL diagnosis in 1997, when he presented swelling supraclavicular and cervical lymph nodes associated with fever, loss of weight and sweaty nights. Histological examination of the cervical lymph node revealed HL, of a mixed cellularity subtype. He received four courses of chemotherapy based on doxorubicin, bleomycin, vinblastine and dacarbazine, along with radiation therapy, but he discontinued the therapy and the follow up.

Physical examination revealed a soft tissue tumor in the right frontal lobe area; neurological examination revealed a spastic paraparesis with anal and vesical sphincter involvement, without sensitive level, bilateral Babinsky and osteotendinous hyperreflexes. His fundoscopic examination was negative for papilledema. The rest of his physical examination was normal.

Received on 17 June 2006; revised 3 November 2006.

Address for correspondence: Dr..Marcelo Corti, Division of HIV/ AIDS Disease, Infectious Diseases F.J. Muñiz Hospital, Puán 381 $2^{\circ} \mathrm{C} 1406 \mathrm{CQG}$, Buenos Aires, Argentina.

E-mail: marcelocorti@ fibertel.com.ar.

The Brazilian Journal of Infectious Diseases 2006;10(6):403-405. C) 2006 by The Brazilian Journal of Infectious Diseases and Contexto Publishing. All rights reserved.
A lumbar puncture was performed and demonstrated cerebrospinal fluid with hyperproteinorraquia $(3 \mathrm{~g} / \mathrm{dL})$, and lymphocytic pleocitosis.

A cranial computed tomography (CT) scan showed a solitary, hypodense, right frontal lobe mass, with contrast enhancement, vasogenic edema and moderate mass effect on the middle line structures. On the right subgaleal plane, near the vertex and adjacent of this lesion, the CT scan revealed an image of soft tissue density (Figure 1). Stereotactic biopsy was performed; histopathological examination demonstrated a lesion composed of a neoplastic proliferation with numerous atypical lymphocytes, scattered eosinophils, rare binucleate lacunar cells and Reed-Sternberg (R-S) cells, which showed immunoreactivity with CD30 and CD15. The histological diagnosis was mixed-cellularity HL. Examining the brain lesion, Epstein-Barr virus (EBV) presence was found in R-S cells; numerous R-S cells showed immunoreactivity for Epstein-Barr virus latent membrane protein type-1 (LMP-1) by immunohistochemistry (IHQ) and Epstein-Barr encoded RNA (EBERs) by in situ hybridization (ISH) in formalin-fixed and paraffin-embedded sections. Special stains were negative for bacteria, fungi, spirochetes and acid-fast bacilli. No granulomas were found.

A magnetic resonance imaging (MRI) of the dorsal and lumbar spine was performed and showed vertebral lesions in the dorsal and lumbar area, hypointense in T1-weighted as a sign of replacement of normal tissue by neoplastic infiltration. Also, paravertebral soft-tissue masses corresponding to lymphadenopathies were found (Figures 2 and 3 ). Histopathological examination of a lumbar vertebral biopsy confirmed infiltration by atypical lymphocytes, corresponding to mixed-cellularity HL. The patient was retreated with systemic chemotherapy support by granulocyte stimulating colony factor without response and died quickly, one month after the admission, due to respiratory complications.

\section{Discussion}

Involvement of the CNS by HL occurs in 0.2 to $0.5 \%$ of all cases in advanced stages of the disease [3]. It is generally a 
Figure 1. CT scan showing a right frontal cortico-subcortical mass with irregular enhancement and soft tissue infiltration.

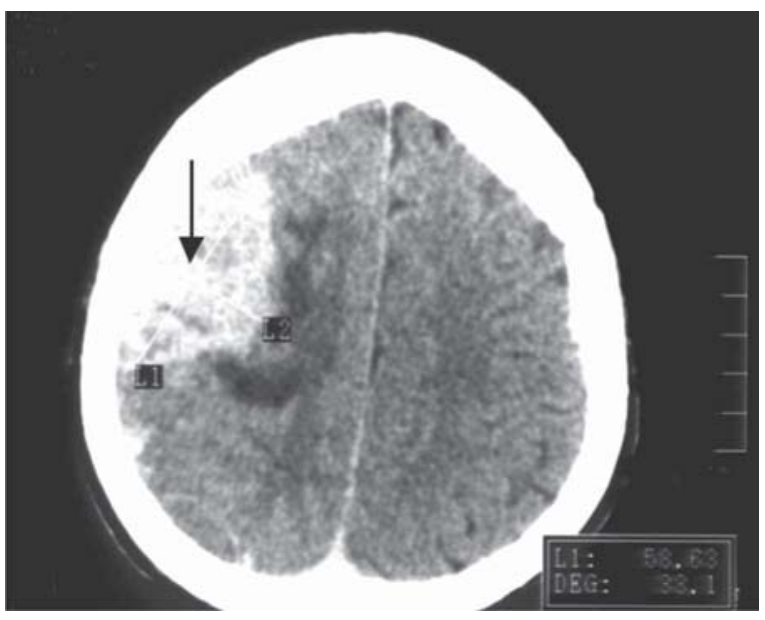

Figure 3. Axial T1 weighted MRI revealing vertebral body infiltration with spinal cord displacement.

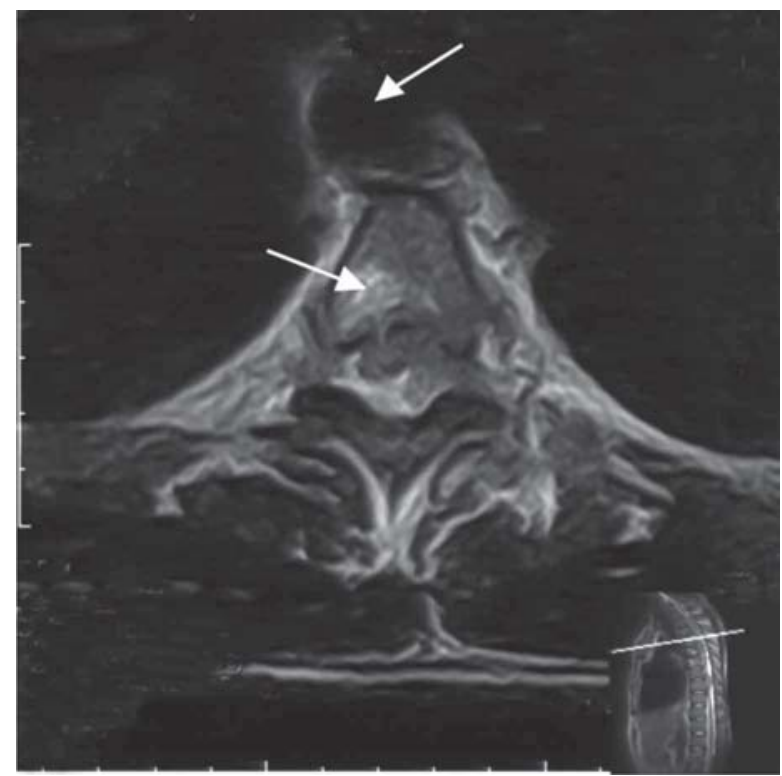

late manifestation and represents a serious and potentially fatal complication for the patient. This complication may occur in patients who are apparently in remission $[1,4]$. Only 25 reports of brain involvement in HL were published from 1954 to 1999 [5]. The mechanism of brain compromise is through either direct tumor extension through the skull bone or meningeal involvement [6,7], or systemic haematogenous dissemination rather than contiguous local invasion [2]. Haematogenous spread is probably the most common mechanism, but we think that in our patient brain involvement
Figure 2. Sagital T2 weighted spinal MRI demonstrating vertebral lesions with neoplastic infiltration of normal tissue.

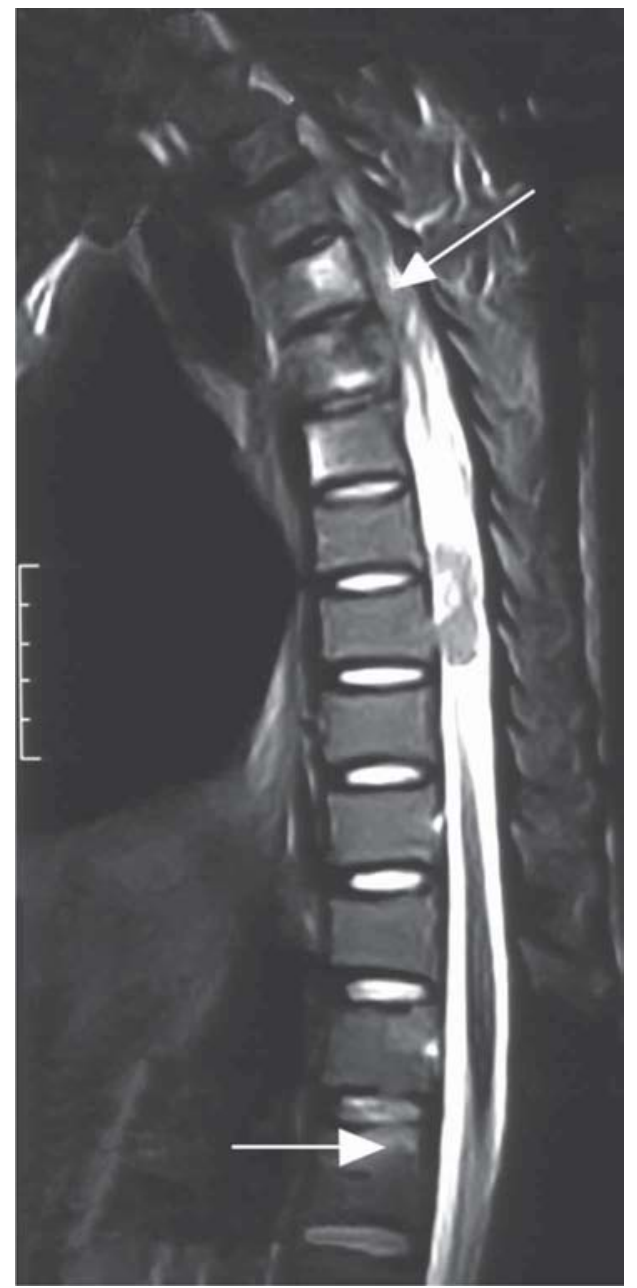

was secondary to the compromise of the skull bone. Also, brain involvement occurs later in the development of HL; it generally occurs many years after evidence of systemic involvement. In our patient, the brain and the spinal cord compromise appeared four years after the onset of nodal involvement.

Most lesions in intracerebral HL have been supratentorial, as in our patient. Cerebral cortex and leptomeninges are most frequently involved, but no area of brain appears to be exempt, and parenchymal metastases have been described, as was seen in our patient $[1,2,6,8]$.

Computed tomography (CT) scan images of intracranial $\mathrm{HL}$ are very variable; the most common aspect is hypodense or hyperdense lesions in the periventricular white matter or basal ganglionic or cerebellar regions, with or without surrounding edema and mass effect [9]. In our patient, CT scan demonstrated a hypodense mass with contrast 
enhancement, mass effect and vasogenic edema in the right frontal lobe, with an adjacent subgaleal image.

Intramedullary spinal cord metastases are uncommon in HL and are associated with a poor prognosis. Only a few cases of this complication have been reported in the medical literature [9]. CT scans show an area of increased attenuation of the spinal cord. MRI has greater sensitivity and specificity than CT in spinal cord evaluation. T2-weighted MRI demonstrate increased signal intensity within the spinal cord, as was seen in our patient. Concomitant vertebral bone involvement has been observed in $30 \%$ to $40 \%$ of cases $[10,11]$.

Vertebral lesions occur most frequently in the dorsal and lumbar spine, as in our patient, and less frequently in the cervical spine. Osteoblastic and osteolytic lesions, patchy sclerosis and "ivory vertebrae" pattern are the most frequent radiological findings. Gouge defects of the anterior border of the vertebrae are the result of erosion by lymph nodes. Lytic and blastic lesions are also frequent. Vertebral collapse is common and paravertebral soft tissue masses, as seen in our patient, are frequently seen $[12,13]$.

Bone marrow involvement is also heterogeneously found and MRI shows a decreased signal intensity in T1-weighted, as in our patient. During the course of HL, only 5\% to $32 \%$ of patients will develop bone marrow involvement; HL infiltration of bone marrow presumes a clinical stage IV disease [14].

In our patient, the diagnosis of HL with brain, lumbar vertebral and spinal cord involvement was suspected based on the medical history of systemic HL with nodal involvement, the clinical manifestations and the radiological findings. The diagnosis was confirmed by the histopathological findings of brain smears obtained by stereotactic biopsy and the biopsy of the lumbar vertebral body.

Epstein-Barr virus is strongly associated with the pathogenesis of HL in AIDS patients. We were able to demonstrate the EBV genome in the R-S cells of the brain biopsy by two techniques, IHQ and ISH. To our knowledge, this is the third published case of HL involving CNS in an HIV-positive patient and the second demonstrating EBV presence [15].

Finally, unusual and aggressive extranodal presentations of HL should alert to the possibility of HIV infection. Whole brain irradiation and systemic chemotherapy remain the treatment of choice for CNS involvement in patients with HL [5,16,17].

\section{References}

1. Sapozink M.D., Kaplan H.S. Intracranial Hodgkin's disease: a report of 12 cases and review of the literature. Cancer 1983;52:1301-7.

2. Scheithauer B.W. Cerebral metastases in Hodgkin's disease. Arch Pathol Lab Med 1979;103:284-7.

3. Blake P.R., Carr D.H., Goolden A.W. Intracranial Hodgkin's disease. Br J Radiol 1986;59:414-6.

4. Klein R., Mullges W., Bendszus M., et al. Primary intracerebral Hodgkin's disease. Am J Surg Pathol 1999;23:477-81.

5. Nakayama H., Tokuuye K., Kagami Y., et al. Brain involvement in Hodgkin's disease: Case reports and review of the literature. Radiation Medicine 2000; 18 : 205-8.

6. Marshall G., Roessmann U., van den Noort S. Invasive Hodgkin's disease of the brain: Report of two new cases and review of the American and European literature with clinical pathological correlations. Cancer 1968;2:621-30.

7. Kaufman G. Hodgkin's disease involving the central nervous system. Arch Neurol 1965;13:555-8.

8. Cuttner J., Meyer R., Huang Y.P. Intracerebral involvement in Hodgkin's disease: A report of 6 cases and review of the literature. Cancer 1979;43:1497-506.

9. Guermazi A., Brice P., de Kerviler E., et al. Extranodal Hodgkin's disease: spectrum of disease. Radiographics 2001;21:161-79.

10. Toprak A., Kodalli N., Alpdogan T.B., et al. Stage IV Hodgkin's disease presenting with spinal epidural involvement and cauda equina compression as the initial manifestation: case report. Spinal Cord 1997;35:704-7.

11. Mascalchi M., Torrelli P., Falaschi F., Dal Pozzo G. MRI of spinal epidural lymphoma. Neuroradiology 1995;37:303-7.

12. Edeiken-Monroe B., Edeiken J., Kim E.E. Radiologic concepts of lymphoma of bone. Radiol Clin North Am 1990;28:841-64.

13. Gaudin P., Juvin R., Rozand Y., et al. Skeletal involvement as the initial disease manifestation in Hodgkin's disease: a review of 6 cases. J Rheumatol 1992;19:146-52.

14. Linden A., Zankovich R., Theissen P., et al. Malignant lymphoma: bone marrow imaging versus biopsy. Radiology 1989; $173: 335-9$.

15. Massarweh S., Udden M.M., Shahab I., et al. HIV-related Hodgkin's disease with central nervous system involvement and association with Epstein-Barr virus. Case Report. Am J Hematol 2003; 72:216-9.

16. Shet A.S., Saba N., Rausch D., Belzer M. Intra-cranial lesions in a patient with Hodgkin lymphoma. Leuk Lymphoma 2004;45:419-22.

17. Hirmiz K., Foyle A., Wilke D., et al. Intracranial presentation of systemic Hodgkin's disease. Leuk Lymphoma 2004:45:1667-71. 